\title{
A Maximization Problem Involving a Fractional Laplace Type Operator
}

\section{Chong Qiu}

Faculty of Mathematics and Physics, Huaiyin Institute of Technology, Huaian, China

\section{Email address:}

qchsuda@163.com

\section{To cite this article:}

Chong Qiu. A Maximization Problem Involving a Fractional Laplace Type Operator. American Journal of Applied Mathematics. Vol. 9, No. 3, 2021, pp. 86-91. doi: 10.11648/j.ajam.20210903.14

Received: May 25, 2021; Accepted: June 4, 2021; Published: June 16, 2021

\begin{abstract}
Fractional Laplacian is an important nonlocal operator which has many applications in different kinds of differential equations. Recently, optimization problems involving the fractional Laplacian have been studied a lot by many authors. However, most of these papers are focusing on the optimization problems related to the first eigenvalue of the equation. Optimization problems related to the energy functional of the equation have not been investigated well enough. In this paper, we are going to study a maximization problem related to the energy functional of an equation involving a fractional Laplace type operator. Firstly, by using suitable variational framework in a fractional Sobolev space, we can show that a fractional equation has a solution which is in fact the global minimum of the corresponding energy functional. Moreover, by using reduction to absurdity we can obtain the uniqueness of the solution of the fractional equation. Then, we focus on a maximization problem related to the equation which takes the energy functional as the objective functional. Finally, by carefully analysing the properties of an arbitrarily choosen minimizing sequence and the tools of the rearrangement theory, we can prove that the maximization problem is solvable.
\end{abstract}

Keywords: Maximization, Fractional Laplace, Rearrangement

\section{Introduction}

Since Burton's theory of rearrangement was established in the papers [1-2], there have been many applications in optimization problems related to different kinds of equations. Cuccu et al studied the optimization problems involving the p-Laplacian in [3-5]. Emamizadeh and Zivari-Rezapour investigated an optimization problem related to a linear elliptic boundary problem in [6]. Emamizadeh and Prajapat obtained the symmetry of the solution of an optimization problem in [7]. Marras used a different approach to prove the solvability of an optimization involving the p-Laplacian in [8]. Qiu et al introduced a new truncation trick to solve two optimization problems involving the p-Laplacian in [9]. Recent years, many authors were interested in the optimization problems involving the nonlocal operator, see for example in [10-16] and the references therein. Qiu et al considered an optimization problem related to the following fractional equation in [10]:

$$
\left\{\begin{array}{l}
-L_{\theta}^{S} u+h(x, u)=f(x), x \in \Omega, \\
u=0, x \in R^{N}-\Omega
\end{array}\right.
$$

where $-L_{\theta}^{s} u$ is a fractional Laplace type operator defined as follows:

$$
-L_{\theta}^{s} u(x)=\int_{R^{N}} \frac{u(x+y)+u(x-y)-2 u(x)}{|y|^{N+2 s}} \theta(y) d y
$$

with $0<s<1$. For $h(x, u)$, it is assumed that $h(x, \cdot)$ is non-decreasing for almost all $x \in \Omega$. This assumption is essential in the arguments of proving the uniqueness of the fractional equation and the solvability of the corresponding optimization problems. In this paper, we are going to study a maximization problem related to the following fractional equation $\left(P_{\lambda, f}\right)$ : 


$$
\left\{\begin{array}{l}
-L_{\theta}^{s} u-\lambda V(x) u=f(x), x \in \Omega, \\
u=0, x \in R^{N}-\Omega
\end{array}\right.
$$

where $V(x) \in L^{\infty}(\Omega), \quad V(x)>0$ and $\lambda$ is a positive parameter. Apparently, here the term $-\lambda V(x) u$ has different monotonicity. Therefore, we need overcome more difficulties. Under suitable assumptions, we can show that the equation $\left(P_{\lambda, f}\right)$ has a solution $u_{f}$ which is in fact the global minimizer of the corresponding energy functional. Moreover, we can obtain the uniqueness of the solution by using reduction to absurdity. Then, we consider the following maximization problem:

$$
\text { (Max) } \quad \inf _{g \in R(f)} \Phi(g)
$$

where $\Phi(g)=\int_{\Omega} g u_{g} d x$ and $R(f)$ is the set of all the rearrangements of $f$ which is defined in details in section 2 .

We organize the present paper as follows. In section 1 , we give the brief introduction. In section 2, some preliminaries are prepared before the main results. Section 3 is devoted to show the solvability of the equation $\left(P_{\lambda, f}\right)$ and the uniqueness of solution of the equation $\left(P_{\lambda, f}\right)$. At last, in section 4 , we show that the problem (Max) has a solution.

\section{Preliminaries}

\subsection{Some Definitions}

We give some definitions here. In the paper, we denote by

$$
I_{f}(u)=\frac{1}{2} \int_{R^{2 N}} \frac{(u(x)-u(y))^{2}}{|x-y|^{N+2 s}} \theta(x-y) d x d y-\lambda \int_{\Omega} V u^{2} d x-\int_{\Omega} f u d x
$$

We say $u \in H^{s}(\Omega)$ is a solution of the equation $\left(P_{\lambda, f}\right)$ if for any $v \in H^{s}(\Omega)$ it holds

$$
\int_{R^{2 N}} \frac{(u(x)-u(y))(v(x)-v(y))}{|x-y|^{N+2 s}} \theta(x-y) d x d y-\lambda \int_{\Omega} V u v d x-\int_{\Omega} f v d x=0
$$

\subsection{Some Lemmas}

Lemma 1 ([17] Theorem 6.5) The embedding of $H^{s}(\Omega)$ into $L^{r}\left(R^{N}\right)$ is continuous for $1 \leq r \leq 2 N /(N-2 s)$ and compact for $1 \leq r<2 N /(N-2 s)$.

Lemma 2 ([2] Lemma 2.1) For any $g \in R(f)$ where $f \in L^{p}(\Omega) \quad$ for some $1 \leq p \leq \infty$ then $g \in L^{p}(\Omega)$ and $\|g\|_{p}=\|f\|_{p}$.

Lemma 3 ([7] Lemma 2.3) Let $f \in L^{p}(\Omega)$ and $g \in L^{p^{\prime}}(\Omega)$ where $1 \leq p \leq \infty, \quad p^{\prime}=p /(p-1)$, then there closure of $R(f)$ in $L^{p}(\Omega)$.

Define
$C$ a positive constant. Suppose $f: \Omega \mapsto R$ is a measurable function defined in a smooth bounded domain in $R^{N}$ with $N \geq 3$. We say $g: \Omega \mapsto R$ is a rearrangement of $f: \Omega \mapsto R$ if and only if for any $t \in R$ it holds that:

$$
\operatorname{meas}\{x \in \Omega: f(x) \geq t\}=\operatorname{meas}\{x \in \Omega: g(x) \geq t\}
$$

where meas $(\cdot)$ denotes the Lebesgue measure of the set. The set of all the rearrangements of $f: \Omega \mapsto R$ is denoted by $R(f) . H^{s}(\Omega)(0<s<1)$ is the fractional Sobolev space defined by:

$$
H^{s}(\Omega)=\left\{u \in L^{2}\left(R^{N}\right): u=0 \text { in } \Omega^{c},\|u\|<\infty\right\}
$$

where

$$
\|u\|=\left(\int_{R^{N}} \int_{R^{N}} \frac{(u(x)-u(y))^{2}}{|x-y|^{N+2 s}} d x d y\right)^{1 / 2}
$$

is the norm of $H^{s}(\Omega) . H^{s}(\Omega)$ is Hilbert space with the inner product

$$
\langle u, v\rangle=\int_{R^{N}} \int_{R^{N}} \frac{(u(x)-u(y))(v(x)-v(y))}{|x-y|^{N+2 s}} d x d y
$$

For $1 \leq p<\infty$, we denote by $\|u\|_{p}$ the usual norm in $L^{p}(\Omega)$ and $\|u\|_{\infty}$ the norm in $L^{\infty}(\Omega)$. The energy functional $I_{f}: H^{s}(\Omega) \mapsto R$ corresponding to the equation $\left(P_{\lambda, f}\right)$ is exists $\hat{f} \in R(f)$ which maximizes the linear functional $\int_{\Omega} h g d x$ relative to $h \in \overline{R(f)}$ where $\overline{R(f)}$ is the weak

Lemma 4 Suppose that $V(x)>0$ and $V(x) \in L^{\infty}(\Omega)$ $\theta: R^{N} \rightarrow(0, \infty), \quad \theta(x)=\theta(-x), \forall x \in R^{N} \backslash\{0\} ; \quad \theta \in L^{\infty}\left(R^{N}\right)$ and there exists $\theta_{0} \in R_{+}$such that $\theta(x) \geq \theta_{0}, \forall x \in R^{N}$.

$$
\lambda_{0}=\inf _{u \in H_{V}}\left\{\int_{R^{2 N}} \frac{|u(x)-u(y)|^{2}}{|x-y|^{N+2 s}} \theta(x-y) d x d y\right\}
$$


where $H_{V}=\left\{u \in H^{s}(\Omega): \int_{\Omega} V|u|^{2} d x=1\right\}$, then

Proof. Obviously, $0 \leq \lambda_{0}<\infty$. Choose $\left\{u_{n}\right\}$ as a minimize $\lambda_{0}>0$. sequence, i.e.,

$$
\int_{\Omega} V\left|u_{n}\right|^{2} d x=1, \int_{R^{2 N}} \frac{\left|u_{n}(x)-u_{n}(y)\right|^{2}}{|x-y|^{N+2 s}} \theta(x-y) d x d y \rightarrow \lambda_{0},
$$

Then there exists a subsequence (still denoted by $\left\{u_{n}\right\}$ ), such that $u_{n}$ weakly converges to $\bar{u} \in H^{s}(\Omega)$. Since $H^{s}(\Omega)$ is compactly embedded into $L^{2}(\Omega), u_{n}$ converges strongly to $\bar{u} \in L^{2}(\Omega)$. Therefore,

$$
\int_{\Omega} V\left|\left(\left|u_{n}\right|^{2}-|\bar{u}|^{2}\right)\right| d x \leq\|V\|_{L^{\infty}} \int_{\Omega}\left|\left(\left|u_{n}\right|^{2}-|\bar{u}|^{2}\right)\right| d x \rightarrow 0
$$

So that,

$$
\int_{\Omega} V\left|u_{n}\right|^{2} d x \rightarrow \int_{\Omega} V|\bar{u}|^{2} d x=1
$$

Since $\theta(x)=\theta(-x), \forall x \in R^{N} \backslash\{0\} ; \theta \in L^{\infty}\left(R^{N}\right)$ and there exists $\theta_{0} \in R_{+}$such that $\theta(x) \geq \theta_{0}, \forall x \in R^{N}$,

$$
\left(\int_{R^{2 N}} \frac{(u(x)-u(y))^{2}}{|x-y|^{N+2 s}} \theta(x-y) d x d y\right)^{1 / 2}
$$

is a norm in $H^{s}(\Omega)$ which is equivalent to $\|u\|$.

Combining with the weakly lower semi-continuity of the norm, we have

$$
\lambda_{0} \leq \int_{R^{2 N}} \frac{\overline{(u}(x)-\bar{u}(y))^{2}}{|x-y|^{N+2 s}} \theta(x-y) d x d y \leq \liminf _{n \rightarrow \infty} \int_{R^{2 N}} \frac{\left(\bar{u}_{n}(x)-\bar{u}_{n}(y)\right)^{2}}{|x-y|^{N+2 s}} \theta(x-y) d x d y=\lambda_{0}
$$

which implies that

$$
\lambda_{0}=\int_{R^{2 N}} \frac{(\bar{u}(x)-\bar{u}(y))^{2}}{|x-y|^{N+2 s}} \theta(x-y) d x d y>0
$$

\section{Main Results}

\subsection{The Existence and Uniqueness of the Equation $\left(P_{\lambda, f}\right)$}

We use the following hypotheses on the function $\theta: R^{N} \rightarrow(0, \infty):$

(H1) $\theta(x)=\theta(-x), \forall x \in R^{N} \backslash\{0\}$;

(H2) $\theta \in L^{\infty}\left(R^{N}\right)$ and there exists $\theta_{0} \in R_{+}$such that $\theta(x) \geq \theta_{0}, \forall x \in R^{N}$.

Proposition 1 Assume that (H1) and (H2) hold,

$$
0<V(x) \in L^{\infty}(\Omega), \quad f \in L^{q}(\Omega), q>\frac{2 N}{N+2 s}, \quad 0<\lambda<\lambda_{0},
$$

where $\lambda_{0}$ is defined in Lemma 4 , then the equation $\left(P_{\lambda, f}\right)$ has a unique solution $u_{f} \in H^{s}(\Omega)$ and

$$
I_{f}\left(u_{f}\right)=\inf _{v \in H^{s}(\Omega)} I_{f}(v)
$$

Proof. Choose arbitrarily $u \in H^{s}(\Omega)$, by the Holder inequality and Lemma 1 we have

$$
\left|\int_{\Omega} f u d x\right| \leq\|f\|_{L^{q}}\|u\|_{L_{q^{\prime}}} \leq C\|u\|
$$

where $1<q^{\prime}:=q /(q-1)<2 N /(N-2 s)$. From $(\mathrm{H} 1)$ and $(\mathrm{H} 2)$ we have

$$
\int_{R^{2 N}} \frac{(u(x)-u(y))^{2}}{|x-y|^{N+2 s}} \theta(x-y) d x d y \geq \theta_{0}\|u\|^{2}
$$

Therefore, we can deduce that

$$
I_{f}(u) \geq \frac{\theta_{0}}{2}\left(1-\frac{\lambda}{\lambda_{0}}\right)\|u\|^{2}-C\|u\|
$$

which implies that $I_{f}$ is a coercive functional. Since we can check by standard arguments that $I_{f}$ is weakly lower 
semi-continuous, $I_{f}$ has a minimizer $u_{f} \in H^{s}(\Omega)$ with

$$
I_{f}\left(u_{f}\right)=\inf _{v \in H^{s}(\Omega)} I_{f}(v) .
$$

By the standard arguments we can show that $I_{f} \in C^{1}\left(H^{s}(\Omega), R\right)$ which implies that $u_{f} \in H^{s}(\Omega)$ is a solution of the equation $\left(P_{\lambda, f}\right)$, i.e.,

$$
\begin{array}{r}
\int_{R^{2 N}} \frac{\left(u_{f}(x)-u_{f}(y)\right)(v(x)-v(y))}{|x-y|^{N+2 s}} \theta(x-y) d x d y-\lambda \int_{\Omega} V u_{f} v d x-\int_{\Omega} f v d x=0 \quad I_{g}\left(u_{g}\right)=\frac{1}{2} \int_{R^{N}} \int_{R^{N}} \frac{\left|u_{g}(x)-u_{g}(y)\right|^{2}}{|x-y|^{N+2 s}} \theta(x-y) d x d y \\
-\frac{\lambda}{2} \int_{\Omega} V\left|u_{g}\right|^{2} d x-\int_{\Omega} g u_{g} d x
\end{array}
$$

holds for any $v \in H^{s}(\Omega)$. Assume that the equation $\left(P_{\lambda, f}\right)$ has another solution $w_{f}$. Then we can easily deduce that

$$
\int_{R^{2 N}} \frac{\left(T_{f}(x)-T_{f}(y)\right)(v(x)-v(y))}{|x-y|^{N+2 s}} \theta(x-y) d x d y=\lambda \int_{\Omega} V T_{f} v d x
$$

for any $v \in H^{s}(\Omega)$ where $T_{f}=u_{f}-w_{f}$. Take $v=T_{f}$ in the above equality and notice that $0<\lambda<\lambda_{0}$ we have

$$
\begin{aligned}
& \int_{R^{2 N}} \frac{\left|T_{f}(x)-T_{f}(y)\right|^{2}}{|x-y|^{N+2 s}} \theta(x-y) d x d y=\lambda \int_{\Omega} V T_{f}^{2} d x \\
& <\lambda_{0} \int_{\Omega} V T_{f}^{2} d x \leq \int_{R^{2 N}} \frac{\left|T_{f}(x)-T_{f}(y)\right|^{2}}{|x-y|^{N+2 s}} \theta(x-y) d x d y
\end{aligned}
$$

a contradiction. Therefore, the equation has a unique solution.

\subsection{The Solvability of the Problem (Max)}

Theorem 1 Assume that $0<V(x) \in L^{\infty}(\Omega), f \in L^{q}(\Omega)$, $q>2 N /(N+2 s), \quad 0<\lambda<\lambda_{0}$, then the problem (Max) has a solution $\hat{f} \in R(f)$, i.e.,

$$
\geq \frac{1}{2}\left(1-\frac{\lambda}{\lambda_{0}}\right) \theta_{0}\left\|u_{g}\right\|^{2}-C\|g\|_{L_{q}}\left\|u_{g}\right\|
$$
sequence, i.e., $g_{i} \in R(f), \forall i \in \mathbf{N}$ and

$$
A=\lim _{i \rightarrow \infty} I\left(u_{i}\right)
$$
of $R(f)$ in $L^{q}(\Omega)$. Then,

$$
\left|\int_{\Omega}\left(g_{i}-\bar{g}\right) u d x\right| \rightarrow 0, i \rightarrow \infty .
$$

From Lemma 2, $\|g\|_{L_{q}}=\|f\|_{L_{q}}$, we can deduce from the above inequality that $A$ is finite. Choose $\left\{g_{i}\right\}$ as a minimizing

where $u_{i}=u_{g_{i}}, I\left(u_{i}\right)=I_{g_{i}}\left(u_{g_{i}}\right)$. Then $\left\{u_{i}\right\}$ is bounded in $H^{s}(\Omega)$ and has a subsequence which still denoted by $\left\{u_{i}\right\}$ that converge weakly to $u \in H^{s}(\Omega)$ and strongly to $u \in L^{q^{\prime}}(\Omega)$ for the compact embedding of $H^{s}(\Omega)$ into $L^{q^{\prime}}(\Omega)\left(1<q^{\prime}=q /(q-1)<2 N /(N-2 s)\right)$. Notice that $\left\|g_{i}\right\|_{L_{q}} \equiv\|f\|_{L_{q}}$, then $\left\{g_{i}\right\}$ is bounded in $L^{q}(\Omega)$ which has a subsequence still denoted by $\left\{g_{i}\right\}$ converge weakly to $\bar{g} \in \overline{R(f})^{q, w}$, where $\left.\overline{R(f}\right)^{q, w}$ is denoted by the weak closure

Combining with the Holder inequality we have

$$
\left|\int_{\Omega}\left(g_{i} u_{i}-\bar{g} u\right) d x\right| \leq\left|\int_{\Omega} g_{i}\left(u_{i}-u\right) d x\right|+\left|\int_{\Omega}\left(g_{i}-\bar{g}\right) u d x\right| \leq\left\|g_{i}\right\|_{L^{q}}\left\|u_{i}-u\right\|_{L^{q^{\prime}}}+\left|\int_{\Omega}\left(g_{i}-\bar{g}\right) u d x\right| \rightarrow 0, i \rightarrow \infty
$$

Since $H^{s}(\Omega)$ is compactly embedded into $L^{2}(\Omega),\left\{u_{i}\right\}$ converge strongly to $u$ in $L^{2}(\Omega)$. It holds then,

$$
\lim _{i \rightarrow \infty} \int_{\Omega} V\left(\left|u_{i}\right|^{2}-|u|^{2}\right) d x=0
$$

Therefore,

$$
A=\lim _{i \rightarrow \infty} I\left(u_{i}\right) \geq \frac{1}{2} \int_{R^{N}} \int_{R^{N}} \frac{|u(x)-u(y)|^{2}}{|x-y|^{N+2 s}} \theta(x-y) d x d y-\frac{\lambda}{2} \int_{\Omega} V|u|^{2} d x-\int_{\Omega} \bar{g} u d x
$$


By Lemma 3, linear functional

$$
l: \overline{R(f)})^{q, w} \mapsto R, l(g)=\int_{\Omega} g u d x
$$

has a maximizer $\hat{f} \in R(f)$,

$$
\int_{\Omega} \bar{g} u d x \leq \int_{\Omega} \hat{f} u d x
$$

We can imply from (1) that

$$
A \geq \frac{1}{2} \int_{R^{N}} \int_{R^{N}} \frac{|u(x)-u(y)|^{2}}{|x-y|^{N+2 s}} \theta(x-y) d x d y-\frac{\lambda}{2} \int_{\Omega} V|u|^{2} d x-\int_{\Omega} \hat{f} u d x
$$

Since $\hat{u}$ is the global minimizer of the functional $I_{\hat{f}}$,

$$
\begin{aligned}
I_{\hat{f}}(\hat{u}) & =\inf _{v \in W_{0}^{s, p}(\Omega)} \frac{1}{2} \int_{R^{N}} \int_{R^{N}} \frac{|v(x)-v(y)|^{2}}{|x-y|^{N+2 s}} \theta(x-y) d x d y-\frac{\lambda}{2} \int_{\Omega} V|v|^{2} d x-\int_{\Omega} \hat{f} v d x \\
& \leq \frac{1}{2} \int_{R^{N}} \int_{R^{N}} \frac{|u(x)-u(y)|^{2}}{|x-y|^{N+2 s}} \theta(x-y) d x d y-\frac{\lambda}{2} \int_{\Omega} V|u|^{2} d x-\int_{\Omega} \hat{f} u d x
\end{aligned}
$$

From the above two inequalities, we have

$$
I_{\hat{f}}(\hat{u}) \leq A
$$

Since $A=\inf _{g \in R(f)} I_{g}\left(u_{g}\right), \hat{f} \in R(f)$,

$$
I_{\hat{f}}(\hat{u}) \geq A
$$

So that,

$$
I_{\hat{f}}(\hat{u})=A
$$

Since $\quad I_{\hat{f}}(\hat{u})=-\frac{1}{2} \Phi(\hat{f}), \quad \Phi(\hat{f})=\sup _{g \in R(f)} \Phi(g) . \quad$ We complete the proof.

\section{Conclusions}

In this paper, we are focusing on the maximization problem:

$$
\text { (Max) } \quad \inf _{g \in R(f)} \Phi(g)
$$

where $\Phi(g)=\int_{\Omega} g u_{g} d x$, and $u_{g}$ is the unique solution of the equation $\left(P_{\lambda, f}\right)$ :

$$
\left\{\begin{array}{l}
-L_{\theta}^{s} u-\lambda V(x) u=f(x), x \in \Omega \\
u=0, x \in R^{N}-\Omega
\end{array}\right.
$$

with the right hand term $f$ replaced by its rearrangement $g \in R(f)$.

Since the term $-\lambda V(x) u$ is no longer non-decreasing with respect to the second variable $u$, the arguments of the uniqueness of the solution of the equation $\left(P_{\lambda, f}\right)$ in [10] are not valid here. By using reduction to absurdity and the tools of the rearrangement theory, we can obtain the uniqueness of the solution of the equation $\left(P_{\lambda, f}\right)$ and prove that the maximization problem (Max) is solvable. To the best of our knowledge, the result obtained here is new.

\section{Acknowledgements}

The author would like to thank the anonymous reviewers for the constructive suggestions. This research was funded by Natural Science Foundation of China grants number 11771319 and 11901418, and Natural Science Foundation of Jiangsu Province grant number BK20170590 and Jiangsu Provincial Government Scholarship for Studying Abroad.

\section{References}

[1] Burton, G. R. (1987) Rearrangements of functions, maximization of convex functionals and vortex rings. Mathematische Annalen, 276: 225-253.

[2] Burton, G. R. (1989) Variational problems on classes of rearrangements and multiple configurations for steady vortices. Ann. Inst. Henri Poincare., 6, 295-319.

[3] Cuccu, F., Emamizadeh, B., \& Porru, G. (2006) Nonlinear elastic membrane involving the $\mathrm{p}$-Laplacian operator. Electron. J. Differential Equations, 49, 1-10. 
[4] Cuccu, F., Emamizadeh, B., \& Porru, G. (2009) Optimization of the first eigenvalue in problems involving the p-Laplacian. Proc. Amer. Math. Soc., 137, 1677-1687.

[5] Cuccu, F., Porru, G., \& Sakaguchi, S. (2011) Optimization problems on general classes of rearrangements. Nonlinear Anal., 74, 5554-5565.

[6] Emamizadeh, B., \& Zivari-Rezapour, M. (2007) Rearrangement optimization for some elliptic equations. J. Optim. Theory Appl., 135, 367-379.

[7] Emamizadeh, B., \& Prajapat, J. V. (2009) Symmetry in rearrangemet optimization problems. Electron. J. Differential Equations, 149, 1-10.

[8] Marras, M. (2010) Optimization in problems involving the p-Laplacian. Electron. J. Differential Equations, 2, 1-10.

[9] Qiu, C., Huang, Y. S., \& Zhou, Y. Y. (2015) A class of rearrangement optimization problems involving the p-Laplacian. Nonlinear Anal., 112, 30-42.

[10] Qiu, C., Huang, Y. S., \& Zhou, Y. Y. (2016) Optimization problems involving the fractional Laplacian. Electronic Journal of Differential Equations, 98, 1-15.

[11] Dalibard, A. L., \& Gerard-Varet, D. (2013) On shape optimization problems involving the fractional Laplacian. ESAIM Control Optim. Calc. Var., 19 (4), 976-1013.
[12] Biswas, A., \& Jarohs, S. (2020) On overdetermined problems for a general class of nonlocal operators. Journal of Differential Equations., 268, 2368-2393.

[13] Bonder, J. F., Ritorto, A., \& Salort, A. M. (2018) Shape optimization problems for nonlocal operators. Advances in Calculus of Variations., 11 (4), 373-386.

[14] Bonder, J. F., Rossi, J. D., \& Spedaletti, J. F. (2018) Optimal design problems for the first $\mathrm{p}$-fractional eigenvalue with mixed boundary conditions. Advanced Nonlinear Studies., 18 (2), 323-335.

[15] Pezzo, L. D., Bonder, J. F., \& Ríos, L. L.(2018) An optimization problem for the first eigenvalue of the $\mathrm{p}$-fractional Laplacian. Mathematische Nachrichten., 291 (4), 632-651.

[16] Bonder, J. F., \& Spedaletti, J. F. (2018) Some nonlocal optimal design problems. Journal of Mathematical Analysis And Applications., 459 (2), 906-931.

[17] Di Nezza, E., Palatucci, G., \& Valdinoci, E. (2012) Hitchhiker's guide to the fractional Sobolev spaces. Bulletin of Mathematical Sciences, 136, 521-573. 